\title{
Orchestration of driving simulator scenarios based on dynamic actor preparation and automated action planning
}

\author{
Zhitao Xiong and Johan Olstam
}

\section{Linköping University Post Print}

\section{Tweet}

N.B.: When citing this work, cite the original article.

Original Publication:

Zhitao Xiong and Johan Olstam, Orchestration of driving simulator scenarios based on dynamic actor preparation and automated action planning, 2015, Transportation Research Part C: Emerging Technologies, (56), 120-131.

http://dx.doi.org/10.1016/j.trc.2015.02.008

Copyright: Elsevier

http://www.elsevier.com/

Postprint available at: Linköping University Electronic Press

http://urn.kb.se/resolve?urn=urn:nbn:se:liu:diva-117059 


\title{
Orchestration of Driving Simulator Scenarios - based on Dynamic Actor Preparation and Automated Action Planning
}

\author{
Zhitao Xiong ${ }^{\mathrm{a}, \mathrm{b}, *}$, Johan Olstam ${ }^{\mathrm{c}, \mathrm{d}}$ \\ ${ }^{a}$ Research Centre for Integrated Transportation Innovation (rCITI), \\ School of Civil and Environmental Engineering \\ The University of New South Wales \\ UNSW Sydney NSW 2052 Australia \\ ${ }^{b}$ Institute for Transport Studies and School of Computing, \\ University of Leeds, Leeds LS2 9JT, United Kingdom \\ ${ }^{c}$ Swedish National Road and Transport Research Institute (VTI), \\ SE-58195 Linköping, Sweden \\ ${ }^{d}$ Linköping University, Department of Science and Technology (ITN), \\ SE-601 74 Norrköping, Sweden
}

\begin{abstract}
In driving simulation, a scenario includes definitions of the road environment, the traffic situation, simulated vehicles' interactions with the participant's vehicle and measurements that need to be collected. The scenarios need to be designed in such a way that the research questions to be studied can be answered, which commonly imply exposing the participant for a couple of predefined specific situations that has to be both realistic and repeatable. This article presents an integrated algorithm based on Dynamic Actor Preparation and Automated Action Planning to control autonomous simulated vehicles in the simulation in order to generate predefined situations. This algorithm is thus able to not only plan driving actions for autonomous vehicles based on
\end{abstract}

\footnotetext{
*Corresponding author. Tel.:+61 (2) 93850396

Email address: xiongzhitao@gmail.com (Zhitao Xiong)
} 
specific tasks with relevant contextual information, but also handle longitudinal transportation of simulated vehicles based on the contextual information in an automated manner. The conducted experiment shows that the algorithm is able to guarantee repeatability under autonomous traffic flow. The presented algorithm can not only benefit the driving simulation community, but also benefit relevant areas, such as autonomous vehicle and in-vehicle device design by providing them with an algorithm for target pursue and driving task accomplishment, which can be used to design a human-vehicle cooperation system in the coming era of autonomous driving.

Keywords: Driving simulators, Scenario Orchestration, Surrounding vehicles, Experimental design, Autonomous vehicle

\section{Introduction}

A driving simulator is a tool for imitating real vehicle driving. Instead of driving on a real road the drive takes place in a virtual world. The driver interface may include a real vehicle cabin or consist of a simpler driver interface with only a steering wheel and pedals. Driving simulators are mainly used to study driving behaviour in different driving contexts, i.e. different combinations of road environment, vehicle characteristic, support system(s), weather condition, etc. Compared to other tools for studying driving behaviour, as field trials and test tracks, driving simulators offer:

1) a risk-free environment;

2) the possibility to study hazardous situations not easily investigated in the real world;

3) the possibility to study new support systems, road environments, etc. 
4) repeatable and consistent scenarios.

The scenario is the key to provide a pre-defined environment that experimenters need a participant to experience. It includes definitions of the road environment, the traffic situation, simulated vehicles' interactions with the participant's vehicle ${ }^{1}$ and measurements that need to be collected. Choreography of scenarios, plays an important role in driving simulation. In general, the scenarios need to be designed in such a way that the research questions to be studied can be answered. This commonly imply exposing the participant for a couple of predefined specific situations which has to be both realistic and repeatable.

Realism in driving simulators is related to the creation of an illusion of real driving (Olstam et al., 2011). Good realism in a driving simulator imply that the human participants make similar decisions in the driving simulator as they would in real driving. For the surrounding traffic point of view, realism imply requirements of adopting sufficient realistic driving behaviour models for the surrounding vehicles, as Papelis and Ahmad (2001) makes the following conclusion with respect to realism in driving simulators: "In our experience with research studies in high-fidelity simulators, users generally focus their evaluation of the model realism towards the richness of the behaviors, not their fidelity.".

Repeatability means that the essential conditions in a scenario should be repeatable in each trial (Fisher et al., 2010). The balance between realism

\footnotetext{
${ }^{1}$ In this research, only one participant or participant's vehicle (simulator) is included in a particular scenario.
} 
and repeatability is difficult and depend on the requirements of the scenario, which often includes high requirements on repeatability for statistical power in the result analysis. As a result, behaviours of each simulated vehicle are more or less always strictly controlled in order to guarantee repeatability. However, even for a strictly pre-defined scenario, unexpected movements of vehicles, including the participant's, may interrupt some of the pre-defined interactions. In addition strictly controlled surrounding vehicles may behave less realistic in some situations.

This article present an approach for combining repeatability and realism of driving simulator experiments by orchestrating scenarios with autonomous surrounding vehicles. The work presented in this article is a further development of the scenario orchestration framework named SOAV (Xiong et al., $2012,2014)$. The framework is enhanced by adding an algorithm for dynamic actor preparation based on the algorithm in Olstam et al. (2011). The enhanced version makes it possible to engage the participant's vehicle actively based on pre-defined tasks and rich behaviours in order to create desired situations for interactions. The ability to create repeatable scenarios is demonstrated by applying the revised framework to a two lane motorway scenario including a braking lead car and an passing lane platoon that keep the participant vehicle from changing lane.

The paper is organised as follows. Section 2 gives an overview of related work on orchestration of driving simulator scenarios. The revised algorithm is presented in section 3. The numerical results are presented in section 4 and the section 5 ends the paper with concluding remarks and future research needs. 


\section{Related Work}

In driving simulation, orchestration of scenarios has been a focus of research since the mid 1990s regarding how scenarios can be described and how to use those descriptions (e.g., Cremer et al. 1995). Generally speaking, the scenario orchestration shares the same idea: have a human experiment designer describe every aspect of the scenario, and then author the scenario to relevant simulated vehicles. This process can define the rules or sequence of actions that the simulated vehicles should follow before or when the scenario is exposed to human participants.

In general, three questions need to be answered in order to orchestrate a particular scenario:

1) which simulated vehicles should be involved in the scenario?

2) how will those simulated vehicles be prepared?

3) how can the scenario be produced?

These three questions can be answered in a single framework or algorithm as discussed in Wassink et al. (2005). However, the framework in Wassink et al. (2005) considered a driving education application in which scenarios and events can be launched in a random order, which is commonly not allowed in research applications. Furthermore, Wassink et al. (2005) do not include any details regarding the underlying algorithms. More common is that researchers put a focus on answering each question with separate algorithms as listed below.

Question one can be called the actor management problem. Simulated vehicles can be pre-defined and fully described beforehand (e.g., Wolffelaar 
et al. (1999)). However, some existing methodologies can recruit simulated vehicles for interactions in real-time. For instance, Kearney et al. (1999) developed a state machine-based scenario orchestration language that does not require specification of the simulated vehicles to be involved in an interaction beforehand, which implies that recruitment can be done online. In Olstam et al. (2011), an actor management algorithm was developed by considering the average speeds of simulated vehicles needed to reach the proposed location for interactions. The simulated vehicle with the least conspicuous speed trajectory was assigned to interact with the participant.

Question two can be called the actor preparation problem and is related to the "realism" part of the scenario, as in this phase simulated vehicles can have some autonomy to prepare themselves. In general, researchers always assume that simulated vehicles will show up in the right place at the right time. In Olstam et al. (2011), some work was done regarding how to prepare simulated vehicles in an inconspicuous manner by adopting a specific speed trajectory towards the required location for interactions. Moreover, the trajectory is calculated by considering the estimated future speed of the simulator driver, which is estimated by considering its current speed and a estimation of its desired speed based on an algorithm presented in Olstam et al. (2008).

Question three can be called the scenario execution problem and is related to three aspects: what are the available actions, how to trigger and schedule the actions. In previous work (e.g., Papelis et al. (2001)), the event-driven mechanism was widely used and can be regarded as pre-conditions for particular actions: conditions must be true before these actions are executed. The adoption of autonomous simulated vehicles, makes it possible to define both 
high-level or low level actions: researchers can change a simulated vehicle's destination and make it plan actions according to that (Leitao et al., 1999) or just change its action speed (Papelis et al., 2001). Moreover, the scheduling of such actions is often crafted by humans in order to specify the execution order of actions or sub-scenarios ( e. g., Leitao et al. (1999)).

None of these questions have covered scenario failures, which may force the simulated vehicles to take part in the failed interaction again. However, this is questionable not only because retries may not be allowed by scenario design or time constraints, but also because it may be impossible technically due to the lack of replanning capability or dynamic environment reconstruction, e.g., creation of new road segments. As a result, some scenario development issues may arise (Papelis et al., 2003), such as the following examples:

- Pre-Run Issues:

- the experimenter describes interaction outcomes without corresponding context and,

- predict the wrong reactions from participants.

- Run-Time Issues:

- participants do not want to be engaged in some interactions and,

- some interactions never happen due to design or system issues.

Pre-run issues suggest that an efficient communication mechanism is lacking. Experimenters may fail to foresee the extra actions from a participant as they focus on outcomes only; the experimenters do not have a general 
picture of the capacities of the scenario orchestration mechanism and the simulation software. This communication problem can make the scenario orchestration process time-consuming and make the scenario liable to run-time issues. Thus, it is important that both experiment designers and programmers have relevant pre-knowledge, which includes, but is not limited to, the capacities of the driving simulator software, the potential pre-conditions and the effects of particular interactions.

Run-time issues invariably cause failures, e.g., a trigger does not fire or participants do not want to be engaged. Those failures are difficult to avoid, but there is a possibility that they can be fixed dynamically without help from humans by automatically re-orchestrating the scenarios, if permitted.

In order to deal with the four issues mentioned above, both run-time and pre-run ones, Xiong et al. $(2012,2014)$ developed a framework called SOAV (Scenario Orchestration with Autonomous simulated Vehicles). SOAV is designed to orchestrate scenarios with autonomous vehicles in driving simulators. SOAV contains two major components, an Ontology for Scenario Orchestration (OSO) and an algorithm named NAUSEA (autoNomous locAl manoeUvre and Scenario orchEstration based on automated action plAnning) encoded in a driver model named SAIL (Scenario-Aware drIver modeL). OSO is used to describe scenarios and relevant driving context to a virtual driver in a formal, context-oriented, programming-independent, logic-based, human-understandable and machine-processable manner. SAIL/NAUSEA is utilised by virtual drivers to carry out Assignments, which are tasks that virtual drivers need to accomplish in order to generate the required interactions with the human participant. Assignments can provide relevant contextual 
information to virtual drivers: the Formation Position, Monitor(s), Success Condition(s), Failure Condition(s), Assignment-action(s) and the measurement from the interaction generated by this Assignment (Xiong et al., 2012, 2014).

SAIL/NAUSEA was developed so that: 1) the simulated vehicles that are controlled by virtual drivers can actively engage the participant to avoid failure and 2) if failures happen, the simulated vehicles can be re-driven by virtual drivers to generate the proposed interactions. Generally speaking, Xiong et al. (2012, 2014) found a way of combining the solutions for the three questions above, i. e., a way of combining the autonomous vehicles with controlled events based on automated action planning according to Assignments with required contextual information. Compared to Leitao et al. (2000), Xiong et al. (2012, 2014) did not make assumption regarding levels of autonomy as committing to Assignment has been the internal motivation of the virtual driver. However, Xiong et al. $(2012,2014)$ did not examine how those contextual information should be achieved, e. g., how the required Formation Position should be reached by the vehicles controlled by the virtual driver. Dynamic actor preparation was investigated in Olstam et al. (2011) and an algorithm for longitudinal transportation of actors to reach a specified start conditions for an assignment were presented. As a result, in this paper, NAUSEA will be enhanced with the actor preparation algorithm from Olstam et al. (2011) in order to be able to do longitudinal transportation of simulated vehicles for Assignments. 


\section{NAUSEA with Actor Preparation Algorithm}

\subsection{General Algorithm Description}

NAUSEA is used to answer the following questions raised by one virtual driver, which is called Smith (Xiong et al., 2012, 2014), based on the information in an Assignment list:

- which simulated vehicle should I control/drive to interact with the participant's vehicle? ("Match Role")

- how should I drive the vehicle there? ("Prepare Actor")

- what do I need to do for the Assignments in order to generate the required interactions and can I finish them in time? ("Finish Assignment")

- if something went wrong, e.g., if the Assignment failed after execution or I am not able to reach one of the trigger conditions (e.g. the required location for the Assignment), what should I do? ("Replanning")

As a result, a virtual driver needs to carry out the following sub-tasks in a scenario:

- Initialize: the virtual driver acknowledges what (s)he should do in the scenario according to the information encoded in the Assignments;

- Match Role ${ }^{2}$ : after initialization, the virtual driver will find a simulated vehicle to control according to the successive Assignment(s);

\footnotetext{
${ }^{2}$ Role Matching in this research has put a focus on local optimization by finding an optimized actor for a single Assignment.
} 
- Prepare Actor: after the vehicle has been identified, the virtual driver will drive the vehicle to the proposed Formation Position and prepare for the oncoming Assignment; this process will be followed by the "Finish Assignment" specified below;

- Finish Assignment: after the virtual driver has formed the required formation around the participant's vehicle, (s)he will then carry out and finish the Assignment;

- Clean Up: after the accomplishment of the Assignment, the virtual driver will clean up the scenario by restoring the vehicle with previous original parameter values, e.g., previous desired speed. This process is always handled by a pre-defined Assignment because by doing so, restored values can be controlled according to specific needs;

- Replanning: if there is any failure after the Initialization, the virtual driver will invoke "Match Role" and find another vehicle to be able continue the experiment, so the virtual driver will go back to the "Prepare Actor" phase even if (s)he finds the same vehicle as before.

This paper will enhance the Regulating procedure in NAUSEA to deal with the "Prepare Actor" sub-task. If using the original sub-procedure of speed adaptation, Smith would guide a simulated vehicle to reach a Formation Position based on a constant acceleration/deceleration rate. For instance, in order to reach the required position and headway, a simulated vehicle can be requested to maintain a deceleration rate to approach the participant's vehicle when it becomes the leader of the participant's vehicle. Another limitation of the current sub-procedure is that it cannot handle 
combined position, headway and speed requirements. In this paper, speed adaptation will be enhanced with the algorithm presented in Olstam et al. (2011) in order to approach the participant's vehicle in a unconscious manner and to allow combined headway and speed constraints. Before we go any further, some essential concepts in SOAV are given below,

- the Sim: the Sim refers to the vehicle dynamics and rendering facility for the simulation. It updates simulated vehicles' states every frame according to each one's autonomous driving behaviours and related parameters, e.g., desired speed. It also updates the participant's vehicle's states according to the driving instructions from the participant. It then update the graphics accordingly to display the driving conditions. Moreover, the Sim needs to broadcast information of all vehicles every frame to Smith (the virtual driver) and receive/execute orders from Smith;

- Smith: Smith, inspired by Agent Smith in the film Trilogy "The Matrix" (Wikipedia, 2011), refers to the virtual driver in SOAV and can also request to change the physical scene, e.g., put a cone on the road;

- Temporal Constraints: there are two types of temporal constraints in this research: metric and precedence. For instance, "action $\alpha$ Before action $\beta$ " is a precedence constraint and "the start time of $\beta$ - the finish time of $\alpha \leqslant 100$ (seconds)" is a metric constraint;

- Ego-vehicle/flock: the vehicle or vehicle flock Smith is controlling are termed as "Ego-vehicle" or "Ego-flock" respectively; 
- Formation: a Formation is a set of pre-defined local relative positions around the simulator driver/participant. Vehicles in driving simulation always interact with the participants at these Formation Positions. For instance, position "L" refers to "Leader". More details can be found in Xiong et al. (2012, 2014);

- Action: a Low-level action is defined as an action that only can be performed in one way, one sequence and by one vehicle/flock;

- Recipe: a Recipe is a set of actions that specifies how to perform a complex/High-Level Action;

- Assignment: an Assignment is a task that the virtual driver needs to accomplish in order to generate the required interactions with the human participant. An Assignment can include: 1) a Formation Position that the Ego-vehicle/flock should be driven to; 2) when to trigger this Assignment (monitors); 3) when the Assignment can be regarded as successful or failed (success/failure conditions); and 4) what to do (Assignment-actions).

\subsection{Regulating in the Enhanced NAUSEA}

Basically, speed adaptation is used to approach the participant's vehicle according to the Formation Position. Let us suppose that the first successive Assignment for the virtual driver is $A_{0}$, which involves a leader vehicle controlled by Smith. In this research, focus has been put on one virtual driver controlling one simulated vehicle.

In Figure 1, the virtual driver is controlling the vehicle $\mathrm{V}$ and its final position/relative distance for $A_{0}$ is illustrated; it is proposed to be the 


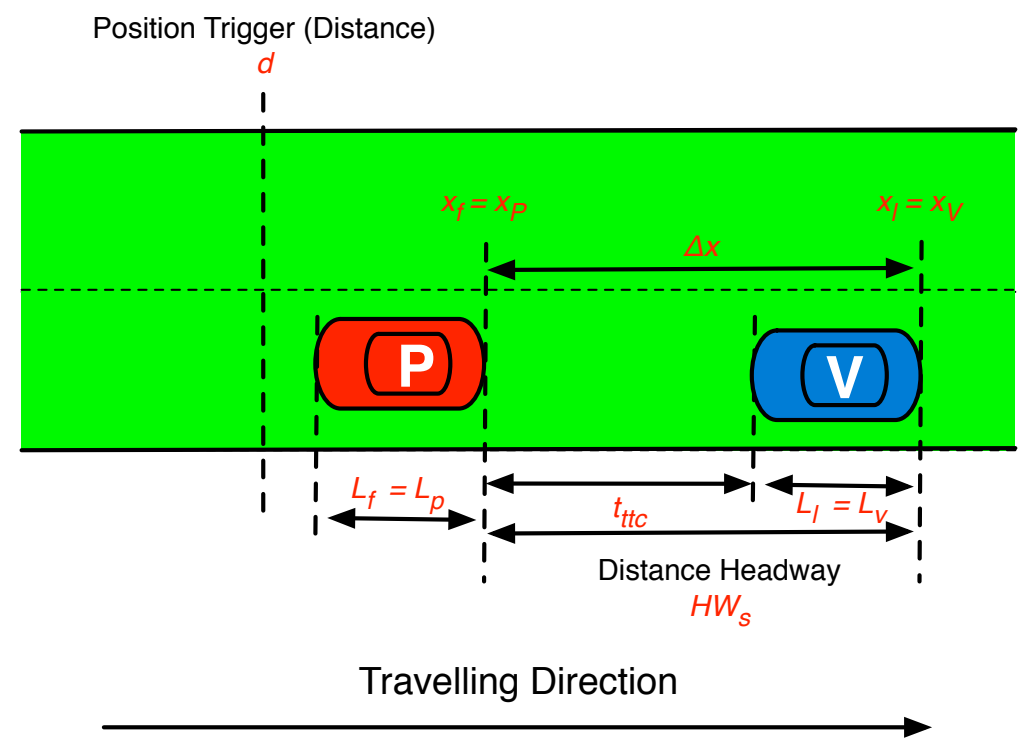

Figure 1: Illustration of Monitor Conditions of $A_{0}$

participant's leader. We denote the lengths of a follower and leader vehicle as $L_{f}$ and $L_{l}$ respectively, and in our case we assume $L_{f}=L_{P}$ and $L_{l}=L_{V}$. The virtual driver should carry out $A_{0}$ when the participant has passed the position $d$, i. e., $x_{P} \geqslant d$ and $d=x_{A_{0}}$. Moreover, its time-tocollision with the vehicle $\mathrm{V}\left(t_{t t c}\right)$ should be large enough to guarantee that the participant's vehicle will not collide with the simulated vehicle V. In the Assignment $A_{0}$ that involves a leader, the distance between the two vehicles $\left(\Delta x=x_{l}-x_{f}=x_{V}-x_{P}\right)$ should be less than the upper distance headway threshold $H W_{s u}$, but greater than the lower distance headway threshold $H W_{s l} . H W_{s u}$ is set to the required distance headway in Monitors and $H W_{s l}$ has been set as $H W_{s u}=50$. Finally, all the information needed to regulate the Ego-vehicle's speed have been provided by the Monitors in Assignments. 
Hence, the Monitors of $A_{0}$ can be informally defined as:

1) As long as the simulator driver reaches or passes $d \mathrm{~m}$ of the road segment;

2) whenever the simulator driver's distance headway to the leader, which is the Ego-vehicle, is less than $H W_{s u} \mathrm{~m}$;

3) whenever the simulator driver's time-to-collision with the leader, which is the Ego-vehicle, is greater than $T T C$ seconds.

Those informal Monitors can be written into OSO (Ontology for Scenario Orchestration) and be fed to SAIL/NAUSEA. If the Assignment will be triggered at $\hat{t}$, the start conditions of $A_{0}$ can be stated as:

$$
\begin{aligned}
x_{P}^{\hat{t}} & \geqslant d \\
H W_{s l}<x^{\hat{t}} & <H W_{s u} \\
t_{t t c}^{\hat{t}} & \geqslant T T C
\end{aligned}
$$

where $x_{P}^{\hat{t}}$ is the position of the participant's vehicle when the Assignment is triggered; $\Delta x^{\hat{t}}$ is the distance between the vehicle and the participant's vehicle when the Assignment is triggered; $t_{t t c}^{\hat{t}}$ is the participant's time-tocollision with the vehicle $\mathrm{V}$ when the Assignment is triggered.

If $v^{\hat{t}}$ is the speed of vehicle $\mathrm{V}$ when the Assignment is triggered and $v_{P}^{\hat{t}}$ is the speed of the participant's vehicle when the Assignment is triggered, then in order to have $t_{t t c}^{\hat{t}} \geqslant T T C$, the following conditions have to be fulfilled: 


$$
\begin{aligned}
x_{P}^{\hat{t}} & \geqslant d \\
H W_{s l}<\Delta x^{\hat{t}} & <H W_{s u} \\
v^{\hat{t}} & \geqslant v_{f}^{\hat{t}}-\frac{\Delta x^{\hat{t}}-L_{l}}{T T C} \\
L_{l} & =L_{V} \\
v_{f}^{\hat{t}} & =v_{P}^{\hat{t}}
\end{aligned}
$$

If $v_{R}$ is the proposed role speed for the vehicle, which should be met when $A_{0}$ is triggered, a set of parameters for the speed adaptation can be derived from the inequalities presented above:

$$
\begin{aligned}
\Delta x_{R} & =D \quad\left(H W_{s l}<D<H W_{s u}\right) \\
v_{R} & =v_{P}^{\hat{t}}-\frac{\Delta x_{R}-L_{V}}{T T C}
\end{aligned}
$$

where $\Delta x_{R}$ is the proposed role distance between the vehicle and the participant when $A_{0}$ is triggered.

To sum up, the virtual driver will guide the vehicle to achieve the following states for $A_{0}$ based on the participant's real-time position $x_{P}$ and speed $v_{P}$, which replaces $v_{P}^{\hat{t}}$ in this research:

$$
\begin{aligned}
\hat{t} & =\left(d-x_{P}\right) / v_{P}+t \\
\Delta x_{R} & =\frac{H W_{s l}+H W_{s u}}{2} \\
v_{R} & =v_{P}-\frac{\Delta x_{R}-L_{V}}{t t c}
\end{aligned}
$$

where $t$ is the current time, so the virtual driver can regulate the vehicle's speed according to the longitudinal actor preparation model presented in Olstam et al. (2011), which give an acceleration rate of: 
$a=\left\{\begin{array}{cl}\frac{v_{a}-v}{t_{c}}, & \text { if } \operatorname{sign}\left(v-v_{a}\right) \neq \operatorname{sign}\left(v_{R}-v_{a}\right) \text { and } t_{c} \leqslant 0.5 \cdot \tilde{t} \\ \frac{v_{a}-v_{R}+v_{a}-v}{0.3 \cdot t}, & \text { otherwise }\end{array}\right.$

where,

sign checks if a number is positive or negative;

$\tilde{t}$ is the time left until the Assignment should start: $\hat{t}-t$;

$v$ is the current speed of the controlled simulated vehicle;

$v_{R}$ is the proposed role speed specified in Equation 13;

$v_{a}$ is the required average speed of the vehicle in order to reach the required position for the Assignment, which can be calculated by adopting $\Delta x_{R}$ and $\hat{t}$ from Equation 12 and 11 respectively:

$$
v_{a}=v_{P}+\frac{\Delta x_{R}-\Delta x}{\tilde{t}}
$$

where $\Delta x$ is the present distance between the vehicle $\mathrm{V}$ and the participant's vehicle.

$t_{c}$ is the time when the vehicle should pass the required average speed in order to reach not only the role speed, but also the proposed start position of the Assignment (see Olstam et al. (2011) for a detailed description). It is calculated as:

$$
t_{c}=\frac{v_{a}-v_{R}}{v-v_{R}} \cdot \tilde{t}
$$


When preparing the vehicle, the virtual driver will use the defined $\hat{t}, \Delta x_{R}$ and $v_{R}$ to regulate the vehicle's speed based on the acceleration rate obtained from Equation 14. When the Monitors of the Assignment are satisfied, the virtual driver will trigger the Assignment even if the values of the three defined parameters are not the same as specified in Equations 11 to 13. The reason is that the virtual driver just needs to make sure that when the Assignment is triggered, the conditions specified in the Monitors, which are represented from Inequalities 1 to 3 , have been satisfied.

In the case that the Formation Position of $A_{0}$ has been specified as a position behind the participant's vehicle, such as "F" or "Follower", $\Delta x$ and $\Delta x_{R}$ will be negative $\left(\Delta x=x_{f}-x_{l}<0\right)$. As a result, the required distance headway will be specified as negative in the first place. The required distance headway will then be rewritten as "bigger than $H W_{s u}$ ". Hence, Equations 4 to 8 will be:

$$
\begin{aligned}
x_{P}^{\hat{t}} & \geqslant d \\
H W_{s l}<\Delta x^{\hat{t}} & <H W_{s u} \\
v^{\hat{t}} & \leqslant v_{l}^{\hat{t}}+\frac{\left|\Delta x^{\hat{t}}\right|-L_{l}}{T T C} \\
L_{l} & =L_{P} \\
v_{l}^{\hat{t}} & =v_{P}^{\hat{t}}
\end{aligned}
$$

As a result, $v_{R}$ will be $v_{P}+\frac{\left|\Delta x_{R}\right|-L_{P}}{T T C}$, where $\Delta x_{R}=\frac{H W_{s l}+H W_{s u}}{2}$ 


\section{Experiment and Results}

\subsection{Introduction}

Numerical experiments has been conducted in order to demonstrate and verify the integration of the actor preparation algorithm from Olstam et al. (2011) with NAUSEA and to demonstrate the recipe of a High-level Ac-

tion "Block". For the experiments a scenario was designed based on Play ${ }^{3}$ three of the scenario used in Olstam et al. (2011), because it involved a Flock of three simulated vehicles plus a lead vehicle ahead of the participant's vehicle. It was a suitable interaction for this experiment as speed adaptation could be used along with a High-Level Action "Block". Furthermore, Durations/Monitors/Failure Conditions/Success Conditions/Action Profiles for each Assignment were different from the original values in Olstam et al. (2011), this in order to make the interactions observable within a visual helicopter view. Changes of those values had no influence on the results of this verification step.

In this experiment, only one road segment was used, so replanning after the failure of Assignments was not allowed. Furthermore, no vehicle restrictions or metric constraints were included and changing Ego-vehicle was allowed.

\footnotetext{
${ }^{3}$ Play has been described by Assignment in this research, so it has been newly defined as a pre-defined situation to which the scenario designers would like the participant to be exposed to. It is generated by Smith according to requirements in the Assignments. A scenario can contain several Plays. A Play may contain several interactions generated by several Actions.
} 


\subsection{Equipment}

A laptop was used to run the VTI's simulation software (the Sim) and Smith. It was equipped with a $2.3 \mathrm{GHz}$ Intel ${ }^{\circledR}$ Core i7 CPU and $16 \mathrm{~GB}$ memory. The threading mechanism in the kernel of the VTI's simulaiton software was updated to make it runnable under OS X. The visualisation system of the simulation software, which is used to present the simulation, was run under OS X using Wine ${ }^{4}$, which is used to run Windows applications on OS X.

\subsection{Experiment}

\subsubsection{Scenario Description}

The scenario consisted of a two-lane motorway with a speed limit of 110 $\mathrm{km} / \mathrm{h}$. It was $12.4 \mathrm{~km}$ long and could take simulator drivers a maximum of 7 minutes to complete (given a reasonable travel speed). At the beginning of the experiment, a dense traffic flow was initiated, so nine simulated vehicles were placed around the participant's vehicle according to the specification in Table 1.

Autonomous simulator drivers were used in this experiment because it is easier to adopt different participant desired speeds using autonomous simulator drivers than using human drivers. Ten autonomous simulator drivers were used and they shared the same behaviour model as the autonomous vehicles in Olstam et al. (2011). The desired speeds of each autonomous simulator driver were set to $105,106,107,108,109,110,111,112,113$ and $114 \mathrm{~km} / \mathrm{h}$ respectively from $5 \mathrm{~km} / \mathrm{h}$ less than the speed limit to $4 \mathrm{~km} / \mathrm{h}$ greater than the

\footnotetext{
${ }^{4}$ http://www.winehq.org
} 
Table 1: Initial Traffic Conditions

\begin{tabular}{|c|c|c|c|c|c|c|}
\hline $\begin{array}{c}\text { Distance to the } \\
\text { Simulator Driver } \\
(\boldsymbol{m})\end{array}$ & $\begin{array}{c}\text { Distance to the } \\
\text { the Start of the Road } \\
(\boldsymbol{m})\end{array}$ & $\begin{array}{c}\text { Vehicle } \\
\text { Number }\end{array}$ & $\begin{array}{c}\text { Autonomous } \\
\text { Simulator } \\
\text { Driver }\end{array}$ & $\begin{array}{c}\text { Vehicle } \\
\text { Model }\end{array}$ & $\begin{array}{c}\text { Lane } \\
\text { (Fast or Slow) }\end{array}$ & $\begin{array}{c}\text { Initial Desired } \\
\text { Speed } \\
(\boldsymbol{m} / \mathbf{s})\end{array}$ \\
\hline 0 & 300 & 0 & Yes & Volvo S40 & Slow & $29.167-31.667$ \\
\hline-297.54243 & 2.45757 & 137 & No & Volvo S40 & Slow & 33.6111 \\
\hline-97.542 & 202.458 & 135 & No & Volvo S40 & Slow & 32.0833 \\
\hline-27.542 & 272.458 & 136 & No & Audi TT & Fast & 31.4722 \\
\hline 100.791 & 400.791 & 132 & No & Volvo S40 & Slow & 30.5556 \\
\hline 300.791 & 600.791 & 133 & No & Toyota Corolla & Slow & 28.1111 \\
\hline 450.791 & 750.791 & 134 & No & Mercedes & Slow & 21.75 \\
\hline 604.124 & 904.124 & 140 & No & Audi TT & Fast & 31.1667 \\
\hline 754.12 & 1054.12 & 138 & No & $\begin{array}{c}\text { Renault } \\
\text { Master }\end{array}$ & Slow & 29.6389 \\
\hline 954.12 & 1254.12 & 139 & No & Mercedes \\
Lorry & Slow & 21.25 \\
\hline
\end{tabular}

speed limit. Without misunderstanding, participants and simulator drivers (DS) are used interchangeably to refer to the simulator vehicle.

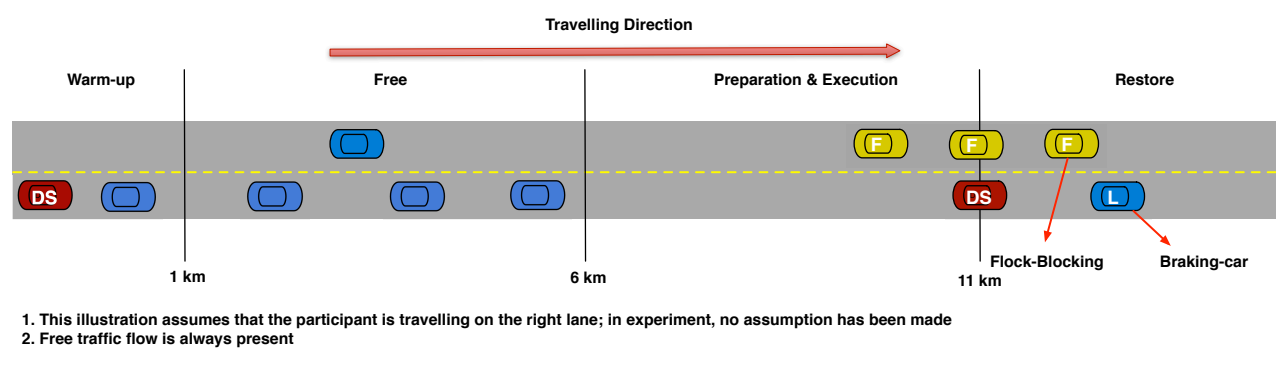

Figure 2: Illustration of the Scenario

As illustrated in Figure 2, this experiment involved four phases: 1) Warmup phase was used to initiate vehicles' speeds; 2) Free phase was a free driving period lasting $5000 \mathrm{~m}$; 3) Preparation and Execution phase involved actor preparation based on the Regulating layer, after which the Assignments were 
performed and 4) Restore Phase was used to restore the traffic flow after the Assignments were executed. Moreover, in the four phases, there were two Assignments for interactions: "Braking-car" and "Flock-Blocking". An extra Assignment was used to indicate when the Role Matching should be invoked.

1) Assignment "Braking-car"

This Assignment involved a simulated vehicle in the Formation Position of "Leader", which is the leader position. When the simulator driver 1) reached $11000 \mathrm{~m}$ of the road segment, 2) reached a relative distance less than 200 meters away from the Ego-vehicle and 3) had a time-tocollision to the leader with a value of INFINITE. The leader should decelerate with an acceleration rate of $-1 \mathrm{~m} / \mathrm{s}^{2}$ and hold this rate for 18 seconds (simulating a car brake down). This Assignment would force the simulator driver to decelerate with the help from the Assignment below, as the simulator driver should be unable to perform overtaking because of a Flock travelling in the adjacent lane.

2) Assignment "Flock-Blocking"

This Assignment shared the same Monitors as the "Braking-car" Assignment and involved a Flock that was used to block the simulator driver when the Assignment "Braking-car" was being carried out. The Assignment-action was a High-Level Action "Block", whose recipe and related information are provided in Section 4.3.2. General speaking, this High-Level Action included several sub-Actions to 1) create a Flock containing three simulated vehicles, 2) clear simulated vehicles that are not an Ego-vehicle/flock before the interaction, 3) maintain the speed of the 
Flock for 18 seconds when the Monitors specified in this Assignment become true and 4) restore the configurations of all simulated vehicles.

3) Assignment "Role-Matching"

This Assignment notified Smith when Role Matching should be invoked. In this experiment, when the driving simulator reached $6000 \mathrm{~m}$ from the start of the road, Smith started to look for an appropriate Egovehicle/flock.

\subsubsection{Additional Information of the Experiment}

Four pre-defined Assignments have been included in the Memory of Smith for the High-Level Action "Block". They are defined to include not only the recipe for "Block", that contains four sub-actions, but also some relevant contextual information:

- Assignment "Create-flock": when the simulator driver reached the position of $6000 \mathrm{~m}$, a Flock would be requested and thus created. The Assignment-action was used to indicate that a Flock containing three simulated vehicles needed to be created. Their initial positions were determined by the fact that the initial average speed were set to be less than $10 \%$ faster than the speed limit, which was set to $110 \mathrm{~km} / \mathrm{h}$;

- Assignment "Clearing": when the simulator driver was $2000 \mathrm{~m}$ away from the proposed Assignment position (11000 $\mathrm{m}$ in the Assignments "Braking-car" and "Flock-blocking"), Smith would start to clear other simulated vehicles that were not members of the Flock or the autonomous simulator driver's lead vehicle. This Assignment was present in order to avoid any simulated vehicles that could interfere with the 
coming interaction. The threshold of $2000 \mathrm{~m}$ was set based on the results from Olstam et al. (2011). The Assignment-action was used to indicate that a clearing process should be invoked. This clearing Action had been implemented directly in the Sim and what Smith needed to do was to send an indicator to invoke the whole process without any details. The clearing Action would set other simulated vehicles that were not Ego-vehicles or members of Ego-flocks with a higher (vehicles that were travelling ahead of the Ego-vehicle/flock, $36 \mathrm{~m} / \mathrm{s}$ ) or lower (vehicles that were behind the Ego-vehicle/flock, $30 \mathrm{~m} / \mathrm{s}$ ) desired speed; This desired speed had proven to be adequate as clearing simulated vehicles in front could be less than 100 meters away in some tests without a sudden change of their acceleration rates;

- Assignment "Maintain-speed": this was used to maintain the speed of the Flock when the Assignment was triggered. Smith would send the Flock ID and an indicator to the Ego-flock including the required speed.

- Assignment "Restore": this was to restore the configurations of all simulated vehicles so that they could return to autonomy with the original desired speed. There was no need to send parameters, Smith would just send an indicator to the Sim and let it carry out restoring activities.

The recipe of the top-Action $\alpha$ was constructed as shown in Figure 3. In this Figure, the Assignment-actions are represented by their parent Assignments' names, e.g., "Create-flock". 


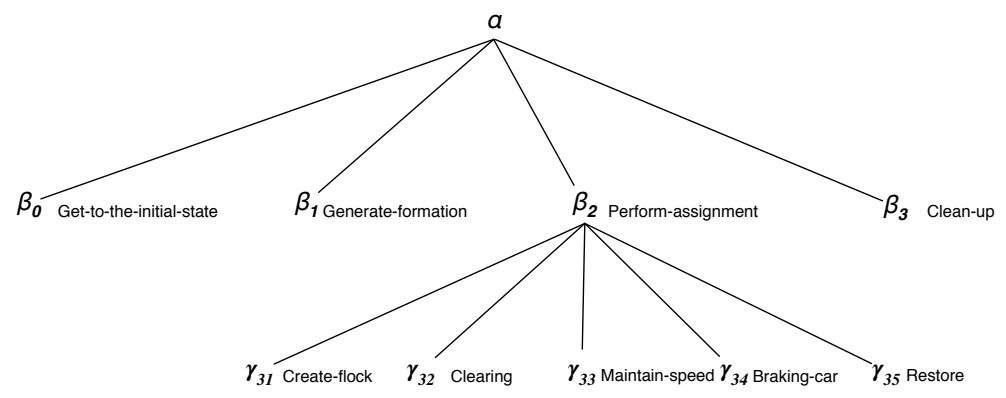

Figure 3: Illustration of Recipe for $\alpha$ ("Perform-scenario")

The precedence constraints were also included:

$\gamma_{31}$ (Create-flock) Before $\gamma_{32}$ (Clearing);

$\gamma_{32}$ (Clearing) Before $\gamma_{34}$ (Braking-car);

$\gamma_{32}$ (Clearing) Before $\gamma_{33}$ (Maintain-speed);

$\gamma_{33}$ (Maintain-speed) Before $\gamma_{35}$ (Restore);

$\gamma_{34}$ (Braking-car) Before $\gamma_{35}$ (Restore);

By using the recipe and constraints above, the generated General Plan included five Assignment-actions as illustrated in Figure 4. Moreover, no metric constraints were specified in this recipe except for the Duration of the Actions $\gamma_{33}$ and $\gamma_{34}$. In this General Plan, Assignment-action $\gamma_{33}$ and $\gamma_{34}$ were parallel.

\subsubsection{Experimental Procedure}

Ten tests were performed sequentially with ten different autonomous simulator drivers, whose desired speeds vary. In each test, the following data 


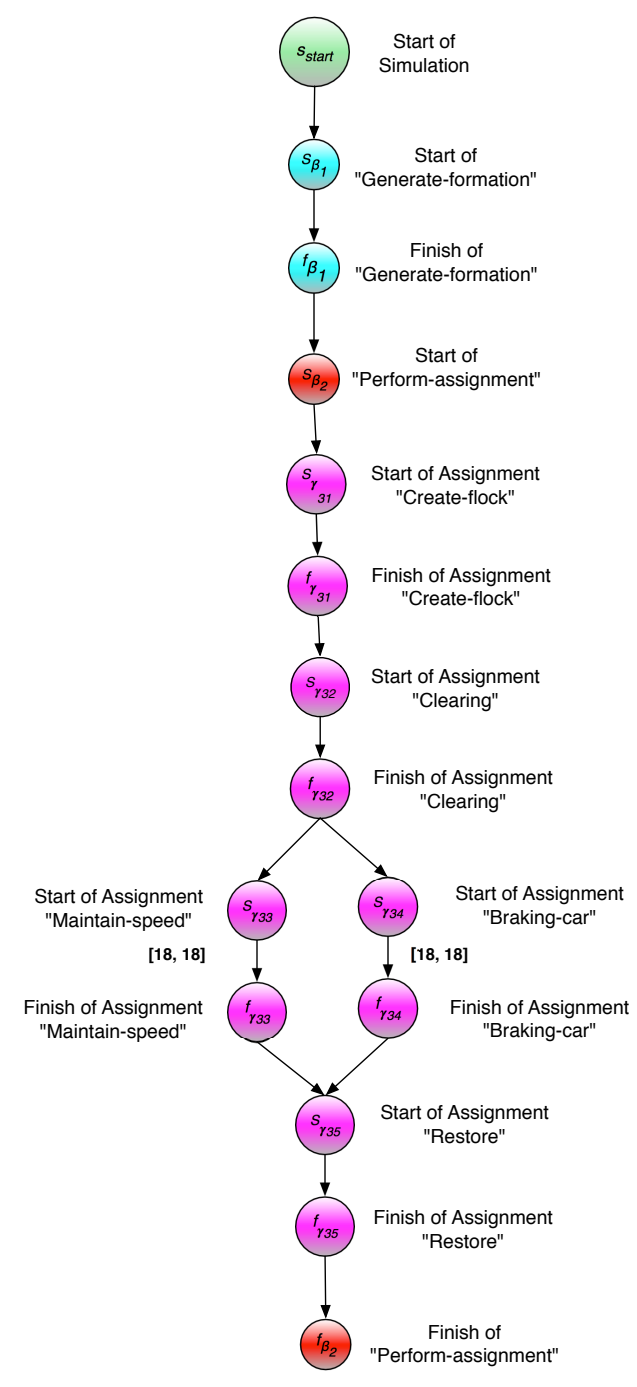

Figure 4: Illustration of General Plan $G r_{\alpha}$ for "Perform-scenario" $(\alpha)$

were recorded:1) Role Matching commands, 2) the start times of the Assignments "Braking-car", "Maintain-speed", "Restore" and 3) positions and speeds of Ego-vehicles and Ego-flocks when orders were executed. 


\subsection{Results}

By utilizing the speed adaptation algorithm derived from Olstam et al. (2011) along with the information from the Assignments, SAIL/NAUSEA successfully constructed the Monitor condition in every test actively and helped NAUSEA to prepare the Ego-vehicle/flock. All the Assignments were also triggered and restored according to Monitors and durations. It is ensured that the scenario could have a leader vehicle and a blocking flock before the required Assignments.

As indicated in Table 2, Smith succeeded in finding a suitable Ego-vehicle for the Assignment "Braking-car". As Role Matching for Flock was not covered in this research, Smith was fed with the Flock information directly in the "Flock-blocking" Assignment.

Table 2: Role Matching Statistics of the Assignment "Braking-Car"

\begin{tabular}{|c|c|c|c|}
\hline $\begin{array}{c}\text { Driver } \\
\text { Number }\end{array}$ & $\begin{array}{c}\text { Desired } \\
\text { Speed } \\
(\mathrm{kmh})\end{array}$ & $\begin{array}{c}\text { Attempts of } \\
\text { Role Matching for } \\
\text { "Braking-car" } \\
\text { (Counts) }\end{array}$ & $\begin{array}{c}\text { Vehicle Found } \\
\text { for "Braking-car" } \\
(I D)\end{array}$ \\
\hline 1 & 105 & 6 & 132 \\
\hline 2 & 106 & 4 & 132 \\
\hline 3 & 107 & 5 & 135 \\
\hline 4 & 108 & 4 & 132 \\
\hline 5 & 109 & 2 & 136 \\
\hline 6 & 110 & 10 & 138 \\
\hline 7 & 111 & 4 & 138 \\
\hline 8 & 112 & 4 & 138 \\
\hline 9 & 113 & 4 & 138 \\
\hline 10 & 114 & 4 & 138 \\
\hline
\end{tabular}


As for the longitudinal transportation of the Ego-vehicle/flock, which is called speed adaptation in NAUSEA, Smith should guarantee that before the execution of the Assignment "Braking-car", the following conditions according to inequalities from 4 to 6 should be met:

$$
\begin{gathered}
x_{P}^{\hat{t}} \geqslant 11000 \\
150<\Delta x^{\hat{t}}<200 \\
t_{t t c}^{\hat{t}} \geqslant \inf
\end{gathered}
$$

As for Ego-flock, the following values were provided directly:

$$
\begin{aligned}
\Delta x_{R} & =50(m) \\
v_{R} & =1.05 \cdot v_{D S}
\end{aligned}
$$

As indicated in Table 3, Smith successfully constructed the conditions according to inequalities from 4 to 5 in order to finish the Assignment. Moreover, as the minimum positive value of $t_{t t c}$ was 466.9077 (driver number 7), inequality 6 was also regarded as fulfilled as the speed difference $\left(v_{p}-v_{V}\right.$, where $v_{P} \geqslant v_{V}$ ) is bigger than $0.5 \mathrm{~m} / \mathrm{s}$ and a collision will not be caused, which is the reason of setting the time-to-collision threshold to INFINITE. In general, the pre-defined time-to-collision threshold, such as INFINITE, is used by Smith to generate a value for regulating the speed of the controlling vehicle, it is not necessary to guarantee that the generated speed is absolutely the same as specified. An error is acceptable if the parent Assignment can be triggered. To sum up, Assignment "Braking-car" was succesfully fired in each test and no participant driver overtook the leader vehicle, thus the Regulating layer was working properly. 
Table 3: Statistics of Start Time, Position and Speed of Ego-vehicle/flock when "Braking-

\begin{tabular}{|c|c|c|c|c|c|c|c|c|}
\hline $\begin{array}{l}\text { Driver } \\
\text { Number }\end{array}$ & $\begin{array}{l}\text { Desired } \\
\text { Speed } \\
(\mathrm{km} / \mathrm{h})\end{array}$ & $\begin{array}{l}\text { Start } \\
\text { Time }\end{array}$ & $\begin{array}{c}\text { Position of } \\
\text { Driver }\end{array}$ & $\begin{array}{l}\text { Speed of } \\
\text { Driver }\end{array}$ & $\begin{array}{c}\Delta x \text { of } \\
\text { Leader Vehicle }\end{array}$ & $\begin{array}{c}\text { TTC of } \\
\text { Leader Vehicle }\end{array}$ & $\begin{array}{c}\Delta x \text { of } \\
\text { Flock Leader }\end{array}$ & $\begin{array}{c}\text { Flock Speed/ } \\
\text { Simulator } \\
\text { Driver's Speed }\end{array}$ \\
\hline 1 & 105 & 380.36 & 11000.2 & 29.1483 & 175.2 & 1832.6360 & 50.1 & 1.0488 \\
\hline 2 & 106 & 377.97 & 11000.2 & 29.4444 & 175.2 & NEGATIVE & 50.1 & 1.0519 \\
\hline 3 & 107 & 375.715 & 11000.2 & 29.7222 & 175.2 & NEGATIVE & 50.1 & 1.0486 \\
\hline 4 & 108 & 374.095 & 11000.2 & 30 & 175.2 & 1836.780 & 50 & 1.0450 \\
\hline 5 & 109 & 363.825 & 11000.3 & 30.2778 & 175.3 & 2782.5397 & 50.1 & 1.0498 \\
\hline 6 & 110 & 360.665 & 11000.2 & 30.5555 & 175 & 19444.4444 & 50.1 & 1.0500 \\
\hline 7 & 111 & 357.62 & 11000.3 & 30.8333 & 167.9 & 466.9077 & 50.1 & 1.0479 \\
\hline 8 & 112 & 354.925 & 11000.2 & 31.1111 & 167.4 & 514.1278 & 50.1 & 1.0478 \\
\hline 9 & 113 & 352.24 & 11000.2 & 31.3889 & 175.2 & 438000 & 50.1 & 1.0520 \\
\hline 10 & 114 & 349.885 & 11000.2 & 31.6666 & 173.1 & 3158.7591 & 50 & 1.0495 \\
\hline
\end{tabular}

\section{Conclusions and future research}

The integration was successful and the results were promising as Assignments can provide relevant contextual information for Regulating, and SOAV was enhanced to handle longitudinal transportation of simulated vehicles for Assignments. As a result, repeatability under autonomous traffic flow can be achieved. However, it should be noted that the application of Regulating could be limited because the estimation of the start time of the Play was not reliable as it was based on real-time participant's speeds. In this case the Ego-vehicle may miss the participants' vehicle and cause failure. More research is therefore needed to estimate the start time of the coming Assignment by anticipating possible reactions from human participants.

Further research is also needed to examine the realistic aspect of the scenario created with the enhanced SOAV, especially regarding Flock control 
in order to manage the members of a Flock with rich behaviours: 1) merging into the Flock; 2) cutting-in to the Flock and 3) leaving the Flock. Some example algorithms can be found in the CyberCar2 project (Ruiz et al., 2008). Those Flock-related algorithms can maybe be adopted by each simulated vehicle and be dictated or requested by Smith.

\section{Acknowledgements}

This experiment received support from HEIF $5^{5}$ and TRENoP ${ }^{6}$. Many thanks also to Jonas Andersson Hultgren and Jonas Jansson (VTI) for help with the integration of SOAV and the VTI driving simulator software.

\section{References}

Cremer, J., Kearney, J., Papelis, Y., 1995. HCSM: a framework for behavior and scenario control in virtual environments. ACM Transactions on Modeling and Computer Simulation (TOMACS) 5 (3), 242-267.

Fisher, D., Rizzo, M., Caird, J., Lee, J. (Eds.), 2010. Scenario Authoring. CRC, Ch. 6, pp. $6.1-6.12$.

Kearney, J., Willemsen, P., Donikian, S., Devillers, F., de Beaulieu, C., Rennes, F., 1999. Scenario languages for driving simulation. In: Proceedings of Driving Simulation Conference,DSC'99. pp. 377-393.

\footnotetext{
${ }^{5}$ Higher Education Innovation Funding, UK

${ }^{6}$ Transport research environment with novel perspectives, Sweden
} 
Leitao, J. M., Sousa, A. A., Ferreira, F. N., 2000. Graphical control of autonomous, virtual vehicles. In: Vehicular Technology Conference Proceedings, 2000. VTC 2000-Spring Tokyo. 2000 IEEE 51st. Vol. 1. IEEE, pp. $507-511$.

Leitao, M., Sousa, A., Ferreira, F., 1999. A scripting language for multi-level control of autonomous agents in a driving simulator. In: Proceedings of Driving Simulation Conference,DSC'99. Vol. 99. pp. 339-351.

Olstam, J., Espié, S., Måardh, S., Jansson, J., Lundgren, J., 2011. An algorithm for combining autonomous vehicles and controlled events in driving simulator experiments. Transportation Research Part C: Emerging Technologies 19 (6), 1185-1201.

Olstam, J. J., Lundgren, J., Adlers, M., Matstoms, P., 2008. A framework for simulation of surrounding vehicles in driving simulators. ACM Trans. Model. Comput. Simul. 18 (3), 1-24.

Papelis, Y., Ahmad, O., 2001. A comprehensive microscopic autonomous driver model for use in high-fidelity driving simulation environments. In: National Research Council (US). Transportation Research Board. Meeting (80th: 2001: Washington, DC). Preprint CD-ROM.

Papelis, Y., Ahmad, O., Schikore, M., 2001. Scenario definition and control for the national advanced driving simulator. In: International Conference on the Enhanced Safety of Vehicles (ESV). SAE International.

Papelis, Y., Ahmad, O., Watson, G., 2003. Developing scenarios to determine 
effects of driver performance: Techniques for authoring and lessons learned. In: Proceedings of Driving Simulation Conference North America,DSC'03.

Ruiz, J. A., de Pedro, T., Isasi, L., Librino, R., Bouraoui, L., Canou, J., González, C., Ducrot, A., November 2008. Decision and Control Primitives for Cooperative Driving Manoeuvres. Cybercar-2 Deliverable 2.1.

Wassink, I., Van Dijk, E., Zwiers, J., Nijholt, A., Kuipers, J., Brugman, A., 2005. Bringing hollywood to the driving school: Dynamic scenario generation in simulations and games. In: Intelligent Technologies for Interactive Entertainment. Springer, pp. 288-292.

Wikipedia, 2011. Agent Smith — Wikipedia, The Free Encyclopedia. Accessed in $10 / 2011$.

URL http://en.wikipedia.org/wiki/Agent_Smith

Wolffelaar, P., Bayarri, S., Coma, I., 1999. Script-based definition of complex driving simulator scenarios. In: Proceedings of Driving Simulation Conference,DSC'99. pp. 353-536.

Xiong, Z., Carsten, O., Jamson, H., Cohn, A. G., 2014. A Task-Driven Framework for Driving Simulation: Scenario Orchestration with Autonomous simulated Vehicles (SOAV). Submitted to ACM Transactions on Interactive Intelligent Systems.

Xiong, Z., Cohn, A. G., Carsten, O., Jamson, H., September 2012. Autonomous local manoeuvre and scenario orchestration based on automated action planning in driving simulation. In: Proceedings of Driving Simula- 
tion Conference Europe 2012. Arts et Métiers ParisTech Paris, France, pp. 233-244. 


\section{List of figure captions}

\section{List of Figures}

1 Illustration of Monitor Conditions of $A_{0} \ldots \ldots$. . . . . . 14

2 Illustration of the Scenario . . . . . . . . . . . . . . . 21

3 Illustration of Recipe for $\alpha$ ("Perform-scenario") . . . . . . . . 25

4 Illustration of General Plan $G r_{\alpha}$ for "Perform-scenario" $(\alpha)$. . 26 


\section{List of table captions}

\section{List of Tables}

1 Initial Traffic Conditions . . . . . . . . . . . . . . . . . 21

2 Role Matching Statistics of the Assignment "Braking-Car" . . 27

3 Statistics of Start Time, Position and Speed of Ego-vehicle/flock when "Braking-car" is Triggered . . . . . . . . . . . . . . . . 29 